\title{
Nonlinear interaction of drift waves with driven plasma currents
}

\author{
Christian Brandt* \\ Max-Planck Institute for Plasma Physics, EURATOM Association, \\ Wendelsteinstraße 1, D-17491 Greifswald, Germany and \\ present address: Jean-Lamour Institute, UMR 7198 CNRS, Dpt P2M, \\ Henri Poincaré University, Nancy IBP 70239, F-54506 Vandoeuvre-lès-Nancy, France \\ Olaf Grulke and Thomas Klinger \\ Max-Planck Institute for Plasma Physics, EURATOM Association, \\ Wendelsteinstraße 1, D-17491 Greifswald, Germany and \\ Ernst-Moritz-Arndt University, D-17489 Greifswald, Germany
}

\begin{abstract}
In a cylindrical magnetized plasma, coherent drift wave modes are synchronized by a mode selective drive of plasma currents. Nonlinear effects of the synchronization are investigated in detail. Frequency pulling is observed over a certain frequency range. The dependence of the width of this synchronization range on the amplitude of the driven plasma currents forms Arnold tongues. The transition between complete and incomplete synchronization is indicated by the onset of periodic pulling and phase slippage. Synchronization is observed for driven current amplitudes, that are some percent of the typical value of parallel currents generated by drift waves.
\end{abstract}

PACS numbers: $05.45 . \mathrm{Xt}, 52.25 . \mathrm{Xz}, 52.30 . \mathrm{Ex}, 52.35 . \mathrm{g}, 52.35 . \mathrm{Kt}, 52.35 . \mathrm{Mw}, 52.35 . \mathrm{Ra}$

\section{INTRODUCTION}

The drift wave instability was intensely studied in the framework of turbulent cross-field transport in magnetic confinement devices $[1,2]$. The fluctuations of density and potential driven by drift waves occur either turbulent or coherent. In certain plasma parameter regimes, drift wave fluctuations are coherent and can be described by a nonlinear oscillating system [3, 4]. Self-sustained nonlinear oscillators generate stable oscillations with a constant amplitude by establishing a balance between energy input and energy dissipation. At small amplitudes the energy dissipation is smaller than the energy input and the oscillation is amplified. At large amplitudes the dissipation is larger and the oscillation is damped. In phase space description stable oscillations form a limit cycle [5]. A paradigm of self-excited oscillations is the van der Pol oscillator [6]

$$
\ddot{x}-\varepsilon\left(1-\beta x^{2}\right) \omega_{0} \dot{x}+\omega_{0}^{2} x=0,
$$

where the parameter $\varepsilon$ determines the degree of the nonlinearity, $\beta$ determines the degree of nonlinear saturation, and $\omega_{0}$ is the angular frequency of the free-running oscillator. Various plasma instabilities have been described as van der Pol-like nonlinear oscillating system [7-10]. In plasma control experiments synchronization of instabilities has been observed [3, 10]. Also drift waves may be seen as a space-time oscillator system [4]. They are driven by the radial density gradient and damped by viscosity. Drift waves have been controlled by closedloop schemes using simple feedback methods with rela-

\footnotetext{
* present address: christian.brandt@lpmi.uhp-nancy.fr
}

tively strong perturbations of the plasma $[11,12]$. Openloop control schemes with predefined space-time signals achieve synchronization of drift waves at smaller perturbation amplitudes [13-15]. In the present paper synchronization of drift waves is investigated using a space-time open-loop control system. The control parameter is a mode-selective drive of currents parallel to the magnetic field. The driven currents match closely the drift mode current patterns. Synchronization ranges and synchronization dynamics of coherent drift waves are systematically examined.

The paper is structured as follows: Section II outlines the experimental device and the spatiotemporal control system. Section III presents the experimental results of the synchronization of coherent drift waves. A brief discussion and a summery are given in Sec. IV.

\section{METHODS}

\section{A. The experimental device}

The experiments were done in the linear magnetized plasma of the helicon device VINETA [16]. It consists of a $4.5 \mathrm{~m}$ long cylindrical chamber with a diameter of $0.4 \mathrm{~m}$. A linear homogeneous magnetic field of $B_{0} \leq 100 \mathrm{mT}$ is generated by a set of 36 magnetic field coils. Argon plasma is produced by a helicon discharge with input powers between $2-3 \mathrm{~kW}$. The maximum densities in the center are about $2 \cdot 10^{19} \mathrm{~m}^{-3}$. In VINETA the drift wave instability has been previously identified [13]. In the region of the maximum density gradient, azimuthally propagating structures of density and potential fluctuations are observed. These are invoked by the drift wave instability. Depending on the magnetic field $B_{0}$ and plasma density $n$, coherent drift waves are destabilized with typ- 


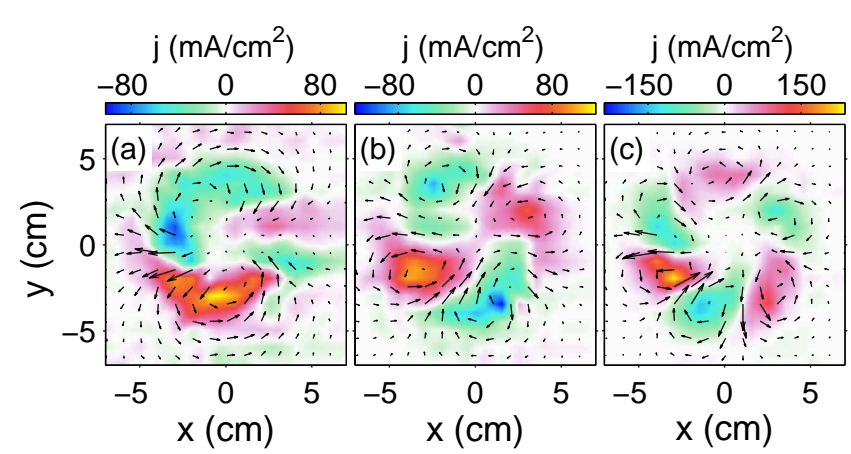

FIG. 1: (Color online) Parallel current filament structure of the drift wave modes (a) $m_{0}=1$, (b) $m_{0}=2$, and (c) $m_{0}=3$ shown in the azimuthal plane. The magnetic field created by the currents is superimposed as arrow vector plots. The length of the arrows correspond to the strength of the magnetic field.

ical frequencies of $f=1-20 \mathrm{kHz}$ and azimuthal mode numbers of $m=1-16$. The plasma currents parallel to the ambient magnetic field $B_{0}$ are a basic feature of the three-dimensional dynamics of drift waves [17]. Fig. 1 shows the parallel current filament structure of the drift modes $m_{0}=1-3$ at a fixed time. The parallel currents are measured using a sensitive $\dot{B}$-probe [18]. Typical values for the "drift wave currents" in VINETA are in the range of $j_{\|} \approx 100 \mathrm{~mA} / \mathrm{cm}^{2}[19]$.

\section{B. Spatiotemporal control system}

Figure 2(a) shows the setup of the space-time openloop control system, the electrostatic exciter. It consists of eight stainless-steel electrodes (size $5 \times 20 \mathrm{~mm}$ ) azimuthally arranged on the circumference of the plasma column at a radius of $4.2 \mathrm{~cm}$. The electrodes are connected to power amplifiers driving currents densities up to $10^{4} \mathrm{~mA} / \mathrm{cm}^{2}$ at frequencies $f=1-20 \mathrm{kHz}$. Figure 2 (b) shows a photo of the electrodes, their connectors to the amplifiers, and the mounting frame. Each electrode is supplied with a predefined sinusoidal voltage signal

$$
U_{n}=U_{d} \sin \left(\omega_{d} t+n \delta\right)
$$

with $\omega_{d}$ being the driver angular frequency. Between the sine signal at electrode number $n$ and electrode number $n+1$, a phase shift

$$
\delta= \pm \frac{2 \pi m_{d}}{k}
$$

is established, where $m_{d}$ is the driver mode number and $k$ the number of electrodes (here $k=8$ ). In this way the electrostatic exciter drives an azimuthally rotating pattern of plasma currents similar to the current filament structure of the corresponding drift wave modes. The driven mode numbers are limited to $m_{d} \leq 3$ owing to the Nyquist-limit $m_{d}<k / 2$. The sign of the phase shift (a)
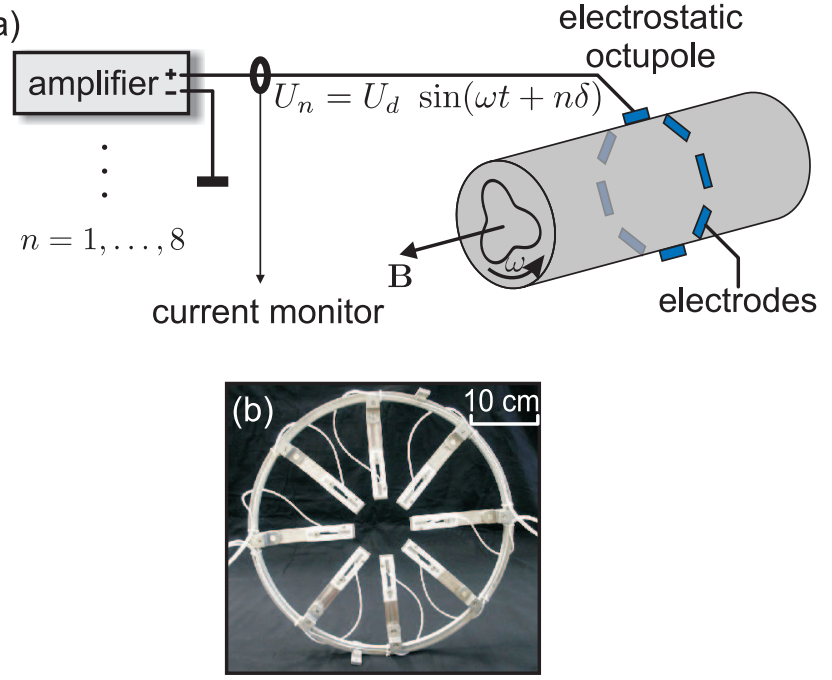

FIG. 2: (Color online) (a) Schematic setup and (b) a photo of the electrostatic octupole exciter.

determines the azimuthal propagation direction of the control pattern. The propagation velocity is varied via the driver frequency $\omega_{d}$.

\section{SYNCHRONIZATION OF COHERENT DRIFT WAVES}

For synchronization of a certain coherent drift wave mode, the exciter is driven with the same mode number and frequency close to the drift wave frequency. Figure 3 shows the frequency spectrum and the space-time diagram of a coherent $m_{0}=1 \mathrm{drift}$ wave mode. The drift wave frequency is $f_{0}=3.3 \mathrm{kHz}$. The peaks at $2 f_{0}, 3 f_{0}$, and $4 f_{0}$ correspond to its higher harmonics. The frequency of the electrostatic exciter is varied in the range from 2.3 to $4.3 \mathrm{kHz}$. In one scan the propagation direction is co-rotating, i.e., in direction of drift wave propagation. In a second scan it is counter-rotating, i.e., opposite to the drift wave propagation. Figure 4 shows the frequency spectra of the scans plotted color coded over the frequency mismatch $\left(f_{d}-f_{0}\right) / f_{0}$ between driver frequency $f_{d}$ and drift wave frequency $f_{0}$. For the upper di-
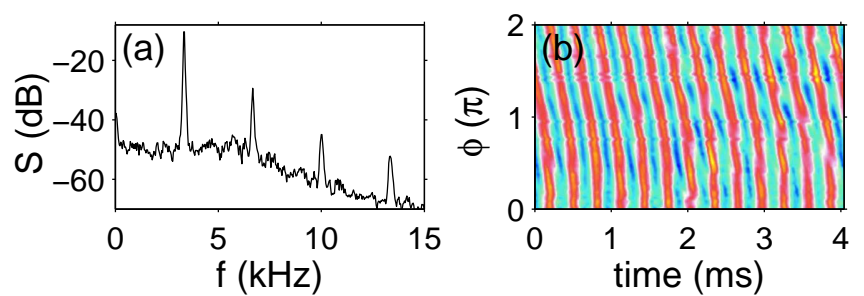

FIG. 3: (Color online) (a) Frequency spectrum and (b) spacetime diagram of a coherent $m_{0}=1$ drift wave mode. 

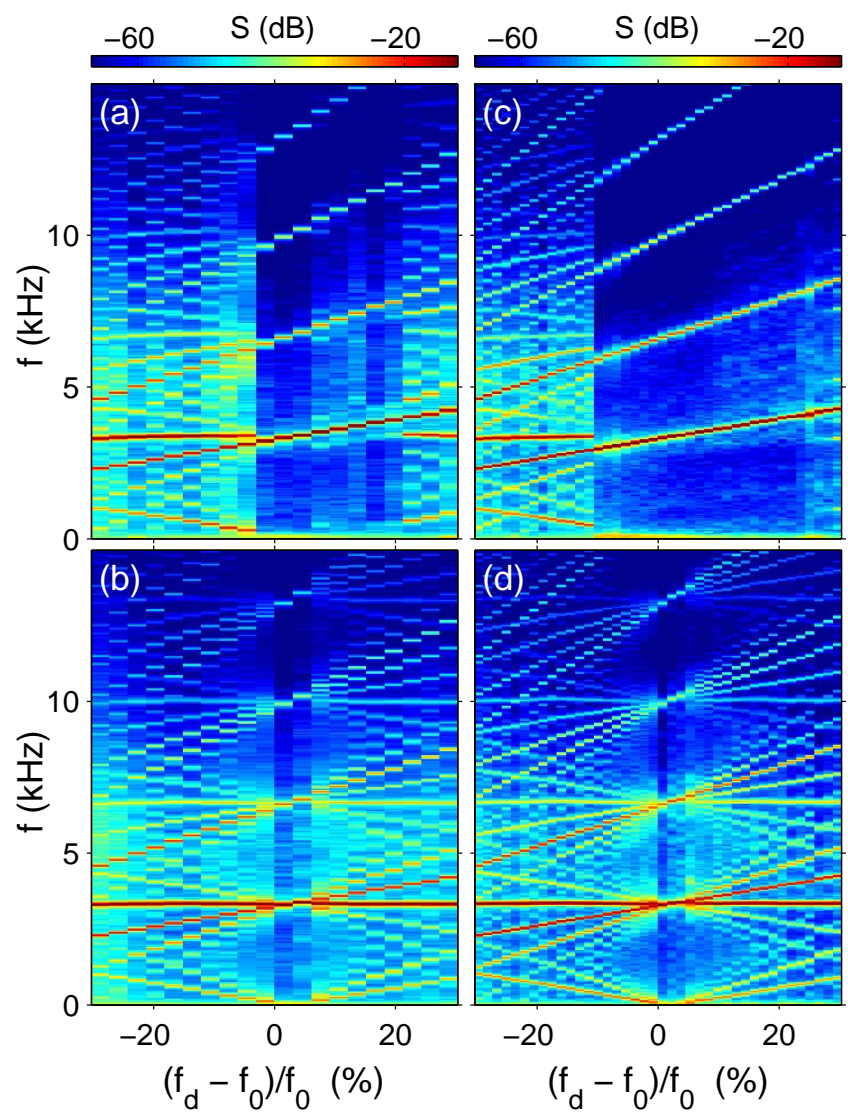

FIG. 4: (Color online) Synchronization of a coherent $m_{0}=1$ drift wave for two different current amplitudes of the electrostatic exciter $\left(j_{d}=79 \mathrm{~mA} / \mathrm{cm}^{2}(\mathrm{a}, \mathrm{b}), j_{d}=94 \mathrm{~mA} / \mathrm{cm}^{2}\right.$ $(\mathrm{c}, \mathrm{d}))$. The exciter is co-rotating in $(\mathrm{a}, \mathrm{c})$ and counter-rotating in (b,d). The exciter frequency is scanned from 2.3 to $4.3 \mathrm{kHz}$ in steps of $100 \mathrm{~Hz}$ in $(\mathrm{a}, \mathrm{b})$ and $50 \mathrm{~Hz}$ in $(\mathrm{c}, \mathrm{d})$.

agrams [Figs. 4(a,c)] the control signal is co-rotating and for the lower diagrams [Figs. 4(b,d)] counter-rotating.

The driver amplitude on the left hand side of the figure is $j_{d}=79 \mathrm{~mA} / \mathrm{cm}^{2}$ and on the right hand side $j_{d}=94 \mathrm{~mA} / \mathrm{cm}^{2}$. During the sweeps the change of the driver frequency can be clearly seen in the shift of the spectral peak from 2.3 to $4.3 \mathrm{kHz}$. At the beginning of the scan [Fig. 4(b)] the drift wave is not synchronized; it is still at its original frequency. The pronounced sideband structure at the difference and sum frequencies result from nonlinear interaction between drift wave and driver signal. If the driver frequency is close to the drift wave frequency, the drift wave locks in, the sidebands disappear and the drift wave starts to track the driver signal. When the drift wave unlocks, the sideband structure reappears. For larger driver amplitudes [Fig. 4(c)] the synchronization range increases. For the counterrotating control signal the synchronization range is significantly smaller [14]. Only if the azimuthal rotation direction of the driven plasma currents is parallel to the drift wave propagation, synchronization over a relatively
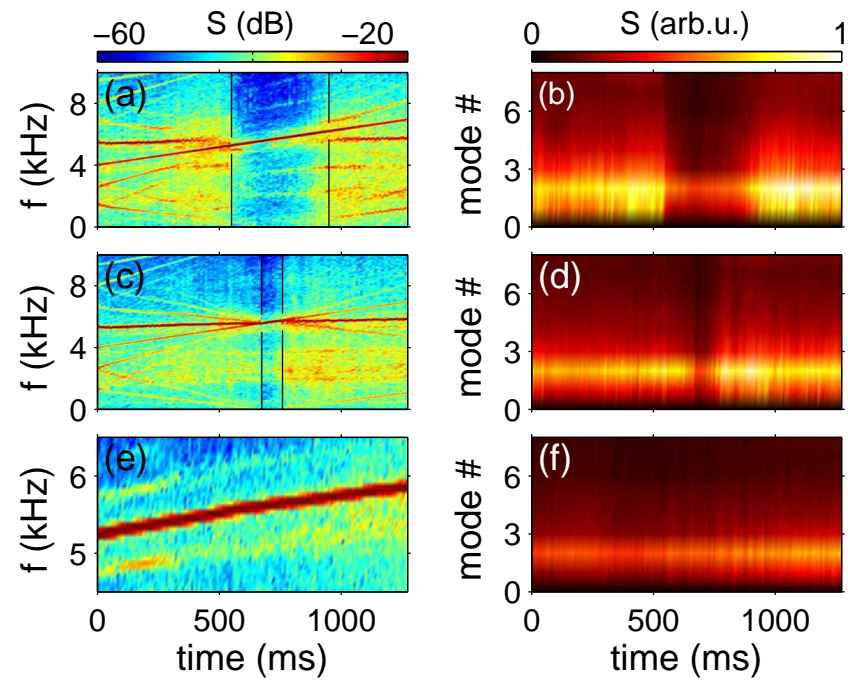

FIG. 5: (Color online) Extraction of Arnold tongues from exciter frequency sweep measurements. Columnwise are shown the frequency and mode-number spectrograms for one plasma pulse. Measurements of the synchronization range for several exciter amplitudes yield an Arnold tongue [Fig. 6].

large frequency range can be achieved. In other words: If the driven current filament pattern is moving slowly or is at rest in the wave frame of the drift mode, synchronization is possible.

For a systematic investigation of the dependence of the synchronization range on driven plasma currents, the synchronization range was measured for different cur-

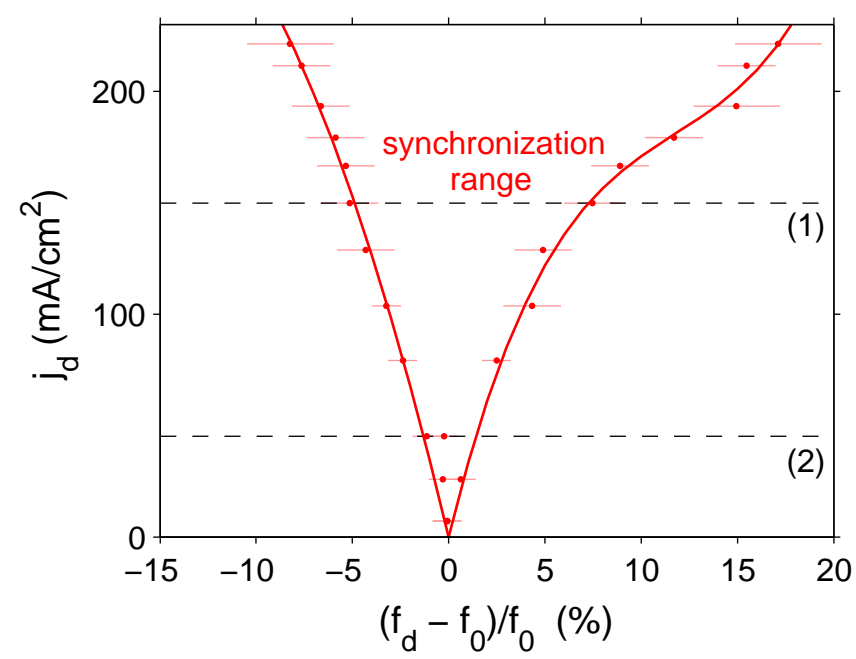

FIG. 6: (Color online) Arnold tongue of a $m_{0}=2$ drift wave mode obtained from exciter frequency sweep measurements. The solid lines are polynomial fits of the third order. The horizontal bars indicate the statistical confidence. The dashed lines (1) and (2) depict the measurements of the synchronization range from the frequency sweeps in Figs. 5(a,b) and $5(\mathrm{c}, \mathrm{d})$. 
rent amplitudes. The driver frequency was linearly swept over the drift wave frequency during one discharge pulse. Figures 5(a-d) show examples of the frequency spectrograms and the mode number spectra for co-rotating exciter frequency sweeps at driver plasma currents $j_{d}=$ $150 \mathrm{~mA} / \mathrm{cm}^{2}(\mathrm{a}, \mathrm{b})$ and $j_{d}=45 \mathrm{~mA} / \mathrm{cm}^{2}$ (c,d). The exciter is driven with a $m_{d}=2$ mode pattern, which matches the drift mode number. For the detection of the synchronization range three criteria must be simultane-
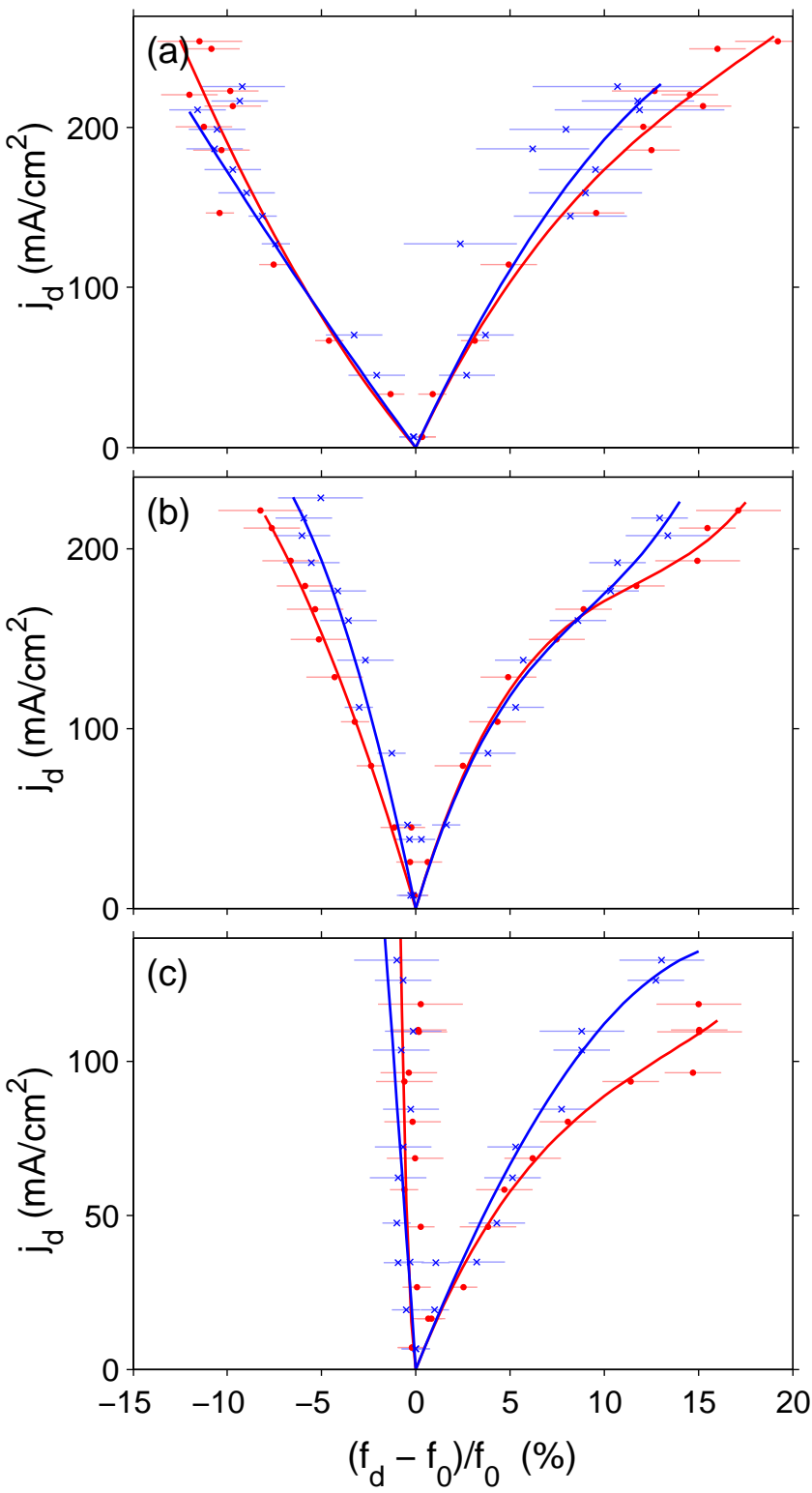

FIG. 7: (Color online) Arnold tongues extracted from frequency sweep measurements for mode numbers (a) $m_{0}=1$, (b) $m_{0}=2$, (c) $m_{0}=3$. The solid lines are polynomial fits of the third order. The red curves marked with red circles correspond to Arnold tongues measured by increasing frequency sweeps, and the curves marked with crosses are measured by decreasing frequency sweeps. The horizontal bars indicate the statistical confidence.

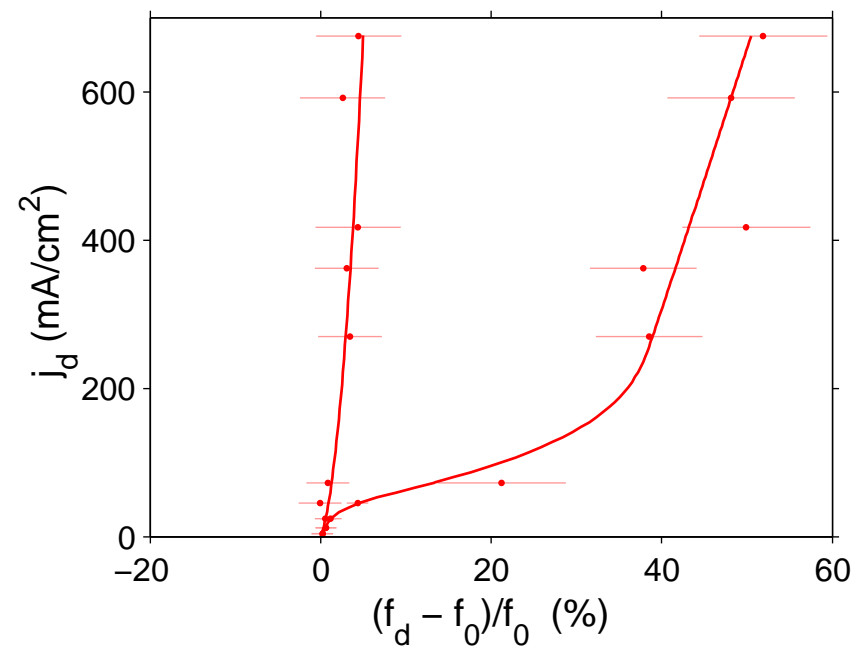

FIG. 8: (Color online) Arnold tongues of a $m_{0}=2 \mathrm{drift}$ wave mode. The solid lines are polynomial fits of the third order. Saturation of the synchronization range is observed at higher exciter current densities. The shaded bars indicate the statistical confidence.

ously satisfied: (1) the drift wave is completely locked to the driver frequency (no sideband structure), (2) the height of the frequency peak is increased, and (3) the mode number is conserved. In this way several sweeps have been recorded for different driver amplitudes. The frequency change of the unperturbed drift wave during the discharge pulse [Fig. $5(\mathrm{e}, \mathrm{f})]$ is also taken into account. The dependence of the synchronization range on the driver amplitude is plotted in Fig. 6. Due to the limited frequency resolution of the frequency sweeps the determination of the locking and unlocking frequency is inaccurate. The horizontal error bars indicate the statistical confidence. At large driver amplitudes the errors are usually larger, since the locking and unlocking region broadens due to the strong nonlinear interaction between driver signal and drift wave [Fig. 5(a)]. The observed increase of the synchronization range with the driver amplitude is known as Arnold tongue [20]. The widths found in Figs. 5(a,c) are indicated by black bars. As a characteristic feature of Arnold tongues both branches, high and low frequency, are touching in one point on the frequency axis at a driver amplitude $j_{d}=0$. For small driver currents, much smaller than the plasma currents caused by the drift waves $j_{d} \ll j_{0} \approx 100 \mathrm{~mA} / \mathrm{cm}^{2}$, the synchronization range increases linearly. If the driver currents approach the drift wave currents $j_{0} \approx 100 \mathrm{~mA} / \mathrm{cm}^{2}$, the width of the Arnold tongue increases overproportionally, which is a typical nonlinear behavior of driven oscillators [20]. The obtained Arnold tongue shows an asymmetry towards larger frequencies at higher driver currents. Figure 7 shows measurements of Arnold tongues for different mode numbers $m=1,2,3$. The Arnold tongues indicated by filled circles are measured with increasing and the ones indicated by crosses with decreas- 

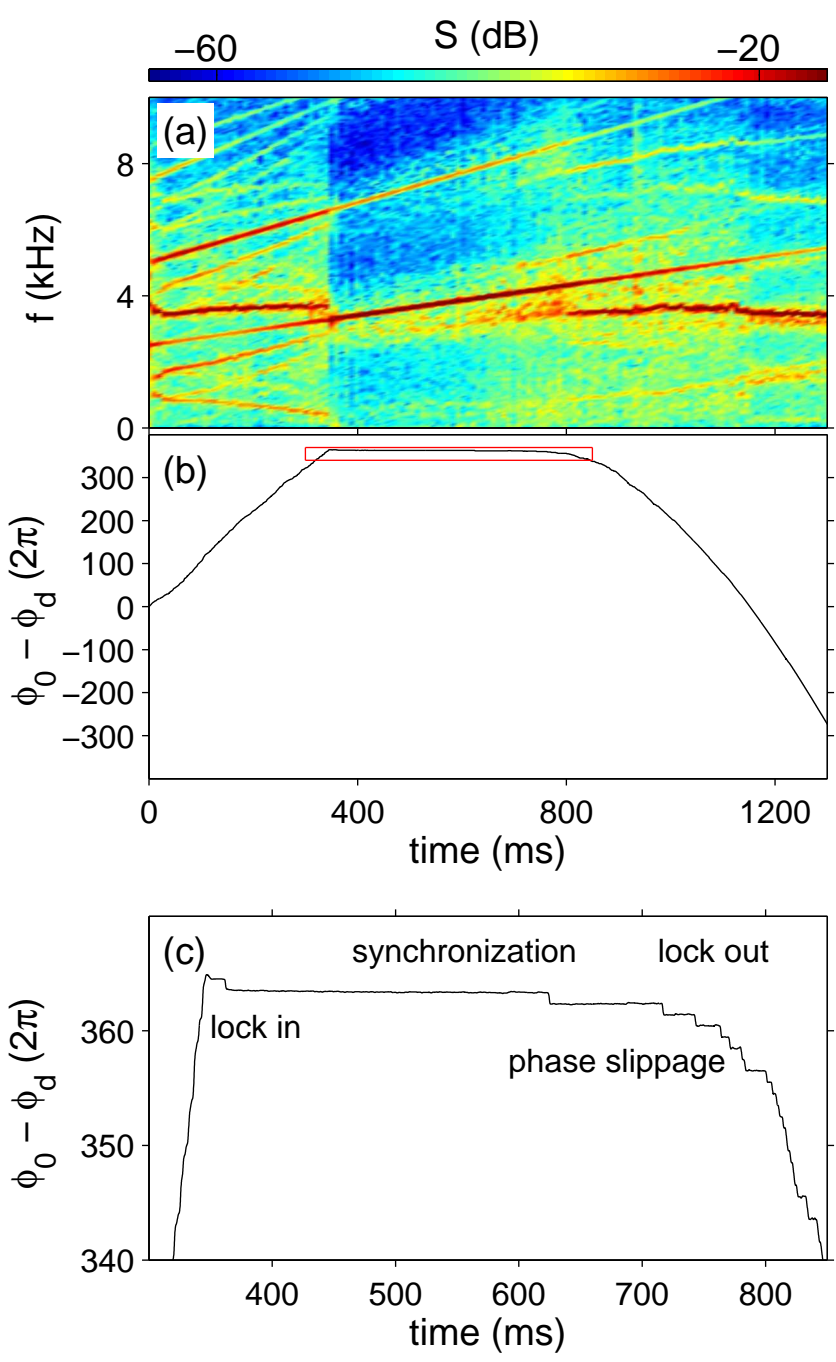

FIG. 9: (Color online) Phase locking during synchronization. When the drift wave locks to the exciter frequency during the upward frequency sweep (a), the drift wave phase is locked (b). The locking region is framed and zoomed in (c).

ing frequency sweeps. Generally the qualitative run of all Arnold tongues show the same tendency as discussed above. The asymmetry towards higher frequencies seems to be stronger for higher mode numbers. For $m=1$ the lowest and highest frequency mismatch is $-10 \%$ and $15 \%$, respectively [Fig. 7], for $m=2$ from $-8 \%$ to $15 \%$, and for the $m=3$ mode from $-2 \%$ to $15 \%$. Distinct hystereris effects of the synchronization range can not be concluded from the increasing and decreasing frequency sweeps, since the difference between them is within the range of the statistical uncertainty. In Figs. 7(a,c) the synchronization range seems to saturate for driver amplitudes higher than the drift wave currents $j_{0}$. Figure 8 shows an Arnold tongue of a $m=2$ drift mode measured for driven plasma currents much larger than $j_{0}$. It shows clear saturation at larger driven plasma currents to both sides of the frequency branches. It is worth to investigate the boundaries of the Arnold tongues in detail. Figure 9 (a) shows the frequency spectrogram of an increasing frequency sweep. The $m_{0}=2$ drift wave mode is present at $3.6 \mathrm{kHz}$. A $m_{d}=2$ driver signal is swept from $f_{d}=2.5-5.5 \mathrm{kHz}$. The drift wave locks at $f=3.3 \mathrm{kHz}$ and unlocks at $f=4.3 \mathrm{kHz}$. Before the occurrence of locking and unlocking, the spectrum around the drift wave peak broadens. The phase difference between driver signal and drift wave is shown in Fig. 9(b). First, the phase difference increases, since the drift wave frequency is higher than the exciter frequency. When the drift wave locks in the phase difference stays constant. When the drift wave unlocks the phase difference decreases, since the exciter frequency is higher than the drift wave frequency. The locking region, indicated by the red frame in Fig. 9(b), is enlarged in Fig. 9(c). At the boundaries of the synchronization range, phase slippage occurs, especially when the drift wave unlocks. For larger frequency mismatches phase slippage occurs more frequently. The phase slippage and the broadening of the peak are evidence for incomplete synchronization and periodic pulling $[10,14,21-23]$.

\section{SUMMARY AND CONCLUSIONS}

In the present paper it has been demonstrated the nonlinear nature of drift wave control by mode-selective driven plasma currents. Different from previous spatiotemporal control experiments on drift waves $[14,15]$ the exciter setup in this paper uses much smaller electrodes $(5 \times 20 \mathrm{~mm})$ with respect to the total plasma size $(l=4 \mathrm{~m}, d=0.4 \mathrm{~m})$. The perturbation by the control signal on the plasma is well localized. Several nonlinear effects, typical for synchronization of oscillating systems, have been found. The driven plasma current pattern is clearly the control parameter. Frequency pulling of coherent drift waves is observed in a certain synchronization range. With increasing driver currents the synchronization range increases thereby forming Arnold tongues. At driver currents lower than the parallel currents generated by drift waves, the Arnold tongues have a linear run. Above the intrinsic drift wave level the slopes develop nonlinearly. At the boundary of the Arnold tongues, in the transition region between uncontrolled and controlled drift wave, periodic pulling is observed. In this region the drift wave and the control signal coexist, but the drift wave is not completely locked. The phase difference between control signal and drift wave shows phase slippage, which has also been observed on other experiments [14]. Drift waves represent a space-time oscillating system, which is nonlinearly saturated by a balance between energy input and energy dissipation. Thus, the natural outcome of space-time control are Arnold tongues, which are useful for predictions of synchronization capabilities of drift wave control systems. 
[1] W. Horton, Rev. Mod. Phys. 71, 735 (1999).

[2] B. D. Scott, New J. Phys. 4, 52 (2002), ISSN 1367-2630.

[3] B. E. Keen and W. H. W. Fletcher, J. Phys. D 3, 1868 (1970).

[4] T. H. Stix, Phys. Fluids 12, 627 (1969).

[5] A. J. Lichtenberg and M. A. Liebermann, Regular and stochastic motion (Springer-Verlag, New York, 1983) 56.

[6] B. van der Pol, Phil. Mag. 3, 64 (1927).

[7] B. E. Keen, Phys. Rev. Lett. 24, 259 (1970).

[8] T. Klinger, A. Piel, I. Axnäs, and S. Torvén, Phys. Scripta 56, 70 (1997)

[9] H. Lashinsky, T. J. Rosenberg, and D. L. Detrick, Geophys. Res. Lett. 7, 837 (1980).

[10] T. Klinger, F. Greiner, A. Rohde, A. Piel, and M. E. Koepke, Phys. Rev. E 52, 4316 (1995).

[11] B. E. Keen and R. V. Aldridge, J. Phys. A 4, 120 (1971).

[12] K. Odajima, Y. Nishida, and Y. Hatta, Phys. Fluids 17, 1631 (1974).

[13] C. Schröder, T. Klinger, D. Block, A. Piel, G. Bonhomme, and V. Naulin, Phys. Rev. Lett. 86, 5711 (2001); C. Schröder, T. Klinger, G. Bonhomme, D. Block, and A. Piel, Contrib. Plasma Phys. 41, 461 (2001).

[14] D. Block, A. Piel, C. Schröder, and T. Klinger, Phys.
Rev. E 63, 056401 (2001);

D. Block, C. Schröder, T. Klinger, and A. Piel, Contrib. Plasma Phys. 41, 455 (2001).

[15] F. Brochard, G. Bonhomme, E. Gravier, S. Oldenbürger, and M. Philipp, Phys. Plasmas 13, 052509 (2006).

[16] O. Grulke, C. M. Franck, T. Klinger, C. Schröder, A. Stark, T. Windisch, and J. Zalach, Contrib. Plasma Phys. 45, 385 (2005).

[17] F. F. Chen, Introduction to plasma physics and controlled fusion (Plenum Press, New York, 1984) 219.

[18] S. Ullrich, Electromagnetic drift waves, Ph.D. thesis, University of Greifswald (2010).

[19] O. Grulke, S. Ullrich, T. Windisch, and T. Klinger, AIP Conf. Proc. 993, 87 (2008).

[20] H. G. Schuster, Deterministic chaos, (VCH, Weinheim, 1989) 158.

[21] R. Adler, Proc. IEEE 61, 1380 (1973).

[22] M. E. Koepke and D. M. Hartley, Phys. Rev. A 44, 6877 (1991).

[23] M. E. Koepke, A. Dinklage, T. Klinger, and C. Wilke, Phys. Plasmas 8, 1432 (2001). 